\title{
Translation elongation and mRNA stability are coupled through the ribosomal A-site
}

\author{
GAVIN HANSON, ${ }^{1,3}$ NAJWA ALHUSAINI, ${ }^{1,3}$ NATHAN MORRIS, ${ }^{2}$ THOMAS SWEET, $^{1}$ and JEFF COLLER ${ }^{1}$ \\ ${ }^{1}$ Center for RNA Science and Therapeutics, Case Western Reserve University, Cleveland, Ohio 44106, USA \\ ${ }^{2}$ Statistical Science Core, Case Western Reserve University, Cleveland, Ohio 44106, USA
}

\begin{abstract}
Messenger RNA (mRNA) degradation plays a critical role in regulating transcript levels in eukaryotic cells. Previous work by us and others has shown that codon identity exerts a powerful influence on mRNA stability. In Saccharomyces cerevisiae, studies using a handful of reporter mRNAs show that optimal codons increase translation elongation rate, which in turn increases mRNA stability. However, a direct relationship between elongation rate and mRNA stability has not been established across the entire yeast transcriptome. In addition, there is evidence from work in higher eukaryotes that amino acid identity influences mRNA stability, raising the question as to whether the impact of translation elongation on mRNA decay is at the level of tRNA decoding, amino acid incorporation, or some combination of each. To address these questions, we performed ribosome profiling of wild-type yeast. In good agreement with other studies, our data showed faster codon-specific elongation over optimal codons and faster transcript-level elongation correlating with transcript optimality. At both the codon-level and transcript-level, faster elongation correlated with increased mRNA stability. These findings were reinforced by showing increased translation efficiency and kinetics for a panel of 11 HIS3 reporter mRNAs of increasing codon optimality. While we did observe that elongation measured by ribosome profiling is composed of both amino acid identity and synonymous codon effects, further analyses of these data establish that Asite tRNA decoding rather than other steps of translation elongation is driving mRNA decay in yeast.
\end{abstract}

Keywords: mRNA decay; translation elongation; codon optimality; decoding

\section{INTRODUCTION}

The major eukaryotic mRNA degradation pathway is initiated by removal of the $3^{\prime}$ poly(A) tail (deadenylation), followed by cleavage of the 5' $7 \mathrm{mGpppN}$ cap (decapping), and exonucleolytic degradation of the mRNA body in the 5'-3' direction (Coller and Parker 2004; Ghosh and Jacobson 2010). Despite being targeted by a common degradation pathway, turnover rates for individual mRNAs differ dramatically, with half-lives in yeast ranging from $<1 \mathrm{~min}$ to $>60$ min (Coller and Parker 2004). While RNA features in untranslated regions have been identified that influence the stability of some mRNAs (Muhlrad and Parker 1992; Lee and Lykke-Andersen 2013; Geisberg et al. 2014), we previously demonstrated that codon optimality (i.e., the balance between tRNA supply and codon demand) influences mRNA decay rates in a more global manner (Presnyak et al. 2015).

The tight coupling between translation status and mRNA turnover in dividing cells has long been appreciat-

\footnotetext{
${ }^{3}$ These authors contributed equally to this work.

Corresponding author: jmc71@case.edu

Article is online at http://www.rnajournal.org/cgi/doi/10.1261/rna. 066787.118.
}

ed (Jacobson and Peltz 1996; Coller and Parker 2004). Specifically, mRNA that is efficiently translated is more stable than mRNA that is translated poorly. The most parsimonious explanation for the link between translation efficiency and mRNA stability is that translation elongation rate is a major driver of mRNA decay rates. Indeed, Saccharomyces cerevisiae ribosome profiling studies have shown that cognate tRNA abundances correlate with translation efficiency (Hussmann et al. 2015; Weinberg et al. 2016), providing support that our observed correlation between codon optimality and mRNA stability may be due to differences in translation kinetics that feedback to the degradation machinery. Importantly, however, the links between codon optimality, translation efficiency, and mRNA stability have not been previously demonstrated on a genome-wide scale. In addition, since work in higher eukaryotes suggests that both A-site decoding and amino acid identity influence mRNA stability (Bazzini

\footnotetext{
(C) 2018 Hanson et al. This article is distributed exclusively by the RNA Society for the first 12 months after the full-issue publication date (see http://rnajournal.cshlp.org/site/misc/terms.xhtml). After 12 months, it is available under a Creative Commons License (Attribution-NonCommercial 4.0 International), as described at http://creativecommons.org/licenses/by-nc/4.0/.
} 
et al. 2016), we sought to determine which of these two aspects of translation elongation correlate more strongly with mRNA stability in S. cerevisae. In this study, we demonstrate that global codon- and transcript-level elongation rate estimates (EREs) inferred by ribosome profiling correlate with mRNA stability. Further analysis of ribosome profiling and of translation efficiency and kinetics of reporter constructs indicate that A-site decoding links translation elongation to mRNA stability in yeast, while the amino acid effects on decay suggested in higher eukaryotes are either weaker or unrelated to translation elongation.

\section{RESULTS}

\section{Codon-specific translation elongation rates correlate with their influence on mRNA stability}

To estimate relative codon-specific elongation rates, we generated a Saccharomyces cerevisiae ribosome profiling data set using cells not pretreated with cycloheximide in order to accurately measure codon-level ribosome dynamics (Gerashchenko and Gladyshev 2014; Hussmann et al. 2015). As expected, a meta-analysis of ribosome protected fragments (RPFs) relative to the start of the coding sequence across all yeast mRNAs shows a clear periodicity of reads in frame with the coding sequence. The well-characterized pileup of reads 12 nucleotides (nt) upstream of the start codon is also present in 28 nucleotide RPFs (Fig. 1A). This is consistent with the start codon residing within the P-site of the ribosome, and we used this positioning to fix the location of the A-site within the reads (Ingolia et al. 2009). Relative per-codon ribosome elongation rates were estimated by utilizing a statistical framework that leverages linear mixed effects modeling of ribosome dynamics across a transcript (see Materials and Methods). This approach allows us to robustly model the error associated with ribosome density estimates and to quantify our confidence in the resulting estimates, while at the same time enabling us to relax arbitrary constraints on the data that we leverage to fit these models. Consistent with previous analyses (Hussmann et al. 2015; Weinberg et al. 2016), we find that our per-codon ribosome EREs correlate well (Pearson $r=0.55, P<10^{-5}$ ) with the tRNA adaptation index (tAl), a measure of relative tRNA availability to the translation machinery of the cell (Fig. 1B; dos Reis et al. 2004). This indicates that higher tRNA abundance is associated with more rapid elongation rates, presumably since the ribosome will more rapidly bind a codon's cognate tRNA when that tRNA is more abundant within the cell.

We next probed the relationship between our estimates of per-codon elongation rate and the codon occurrence to mRNA stability correlation coefficient (CSC), a measure of how individual codons contribute to the stability of mRNA transcripts (Presnyak et al. 2015). Consistent with the corre- lation previously observed between tAl and CSC values (Presnyak et al. 2015), we find a significant and positive relationship between ERE and CSC, both at the level of individual codons $\left(r=0.45[0.22,0.63], P<10^{-3}\right.$; Fig. $\left.1 C\right)$ and when elongation rates and CSC values are averaged across each mRNA (Pearson $r=0.61[0.59,0.63], P<10^{-16}$; Fig. 1D). This latter analysis shows that mRNAs enriched in destabilizing codons are highly enriched in codons that facilitate slow ribosomal elongation.

Having shown a strong global relationship between ribosome elongation and the influence of codons on mRNA stability using our own data, we next sought to expand our analysis to examine this relationship using other ribosome profiling data sets. To this end, we analyzed ten $S$. cerevisiae ribosome profiling data sets generated without the use of cycloheximide (Supplemental Table S1; Cai and Futcher 2013; Gerashchenko and Gladyshev 2014; Guydosh and Green 2014; Jan et al. 2014; Lareau et al. 2014; Pop et al. 2014; Williams et al. 2014; Nedialkova and Leidel 2015; Young et al. 2015; Weinberg et al. 2016) using the same analysis pipeline as our data. While the final normalized EREs varied across data sets, there is a clear partitioning of codons by elongation rate that is consistent across most of the data sets analyzed (Supplemental Fig. S1). A meta-analysis framework was adopted to estimate the true relationship between ERE and CSC values by aggregating the results obtained for each data set into a population-level estimate of this relationship (Fig. 2A). This meta-analysis demonstrates that the body of generated ribosome profiling data in S. cerevisiae, considered in aggregate, is consistent with a significant relationship between per-codon EREs and each codon's influence on mRNA stability $\left(r_{\text {Aggregate }}=0.45[0.34,0.55]\right.$, $P<10^{-4}$ ).

To test whether the observed codon effects are specific to codons located in the A-site of the ribosome, we repeated our analysis for a range of positions relative to the Asite, including the E-site and $\mathrm{P}$-site, as well as regions further upstream and downstream of these sites (Fig. 2B). This allows us to test where the observed relationship between codon identity and transcript stability (CSC) occurs within the ribosome footprint. Only when codons are located in the A-site does the observed relationship between codon-specific EREs and CSCs correlate maximally, consistent with previous observations that codon identity within the A-site is uniquely associated with tAl (Weinberg et al. 2016).

\section{Ribosomal A-site decoding mediates the link between elongation rate and mRNA stability}

The codon stability coefficient (CSC) is only an estimate of how codon content may be associated with transcript stability. More directly relevant is the relationship between the average EREs across a transcript and that transcript's 
A
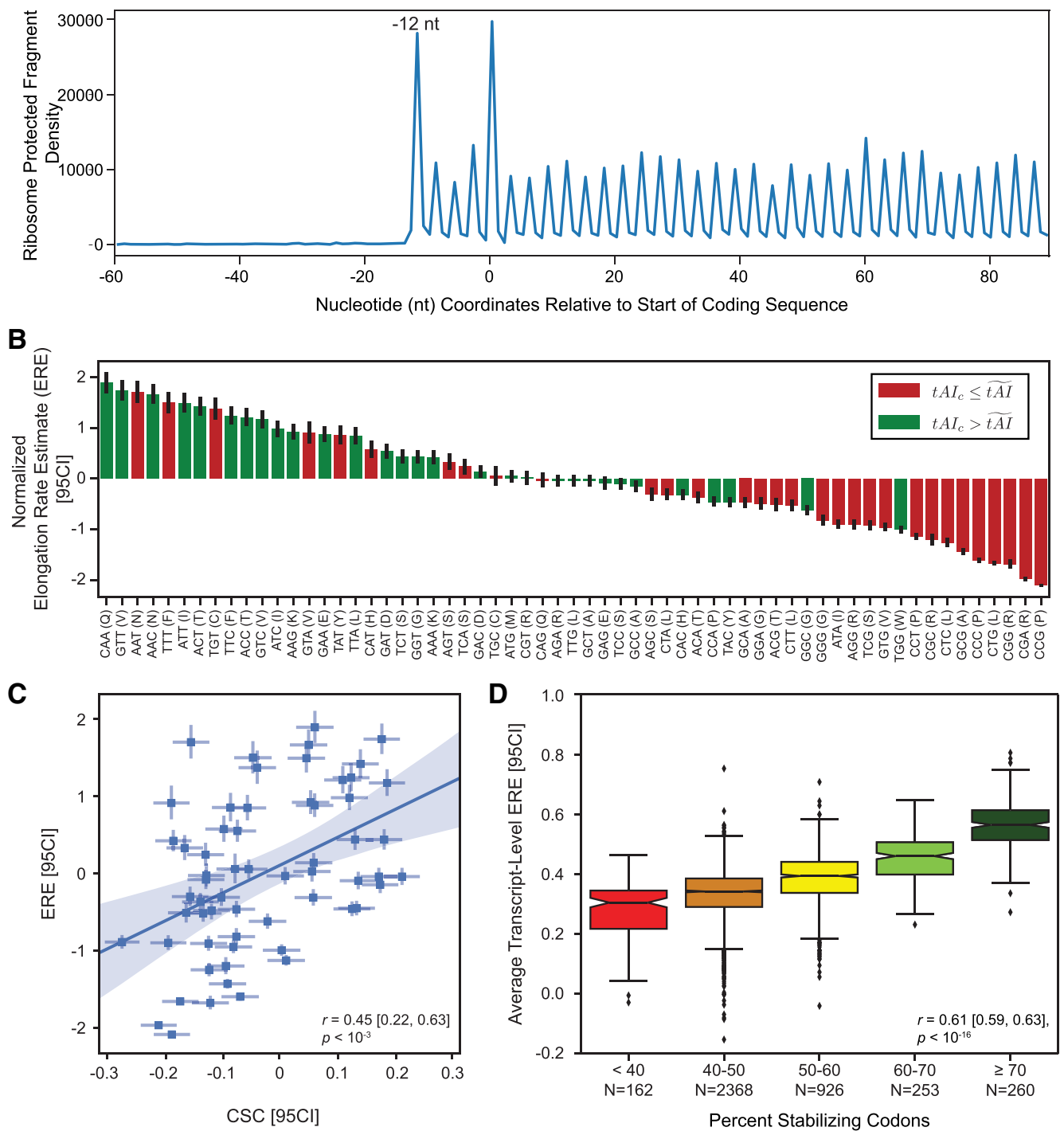

FIGURE 1. Codon-specific translation EREs correlate with codon influence on mRNA decay. (A) Metagene analysis of $28 \mathrm{nt}$ RPFs, relative to the first nucleotide of the coding sequence (position $=0$ ). Read positions are counted based on the $5^{\prime}$ end of the RPFs. $(B)$ Normalized per-codon translation EREs, ordered from fastest to slowest. The EREs for each of the 61 codons have been standardized to have a mean of 0 and a variance of 1 . Error bars represent the $95 \%$ confidence intervals $(95 \mathrm{Cl})$ for the estimate. We have colored each codon to reflect a codon-specific tAl value $\left(\mathrm{t} A \mathrm{I}_{\mathrm{c}}\right.$ ) above (green) or below (red) the median $\mathrm{tAl}$. (C) The best-fit line describing the relationship between normalized per-codon EREs and codon stability coefficients (CSCs), a measure of the influence of codons on mRNA stability. This relationship takes into account the uncertainty in the estimates of elongation rates and CSCs to arrive at a more robust estimate of the overall error in the correlation between these values. Uncertainties in both ERE and CSC values are included in the plot as the $x$-and $y$-axis $95 \%$ confidence intervals $(95 \mathrm{Cl})$, respectively. The shaded region represents the $95 \%$ confidence intervals for the relationship between ERE and CSC. (D) Box plots showing the distribution of average transcript-level normalized EREs associated with the specified levels of percent stabilizing codons within mRNAs globally. Percent stabilizing codons reflects the proportion of codons in an mRNA with a CSC value greater than 0 . Average transcript-level EREs are obtained by averaging per-codon EREs across the entire coding sequence of an mRNA. Notches reflect the standard error of the overall average EREs within each bin. Bin intervals are closed on the left and open on the right.

stability, as determined by mRNA half-life (HL) measurements. Using published mRNA HL data (Presnyak et al. 2015), we globally assessed the relationship between average ERE across a transcript and that transcript's HL. We find a significant and positive relationship between average EREs and transcript $\mathrm{HL}$ in our data $(r=0.28[0.25$,
0.31], $P<10^{-16} ;$ Fig. 3A) as well as in all data sets analyzed (Fig. 3B; red).

It has long been established that tRNA decoding is not the only step that can influence ribosome elongation dynamics. Different amino acids vary in the speed at which they are incorporated into a nascent polypeptide 

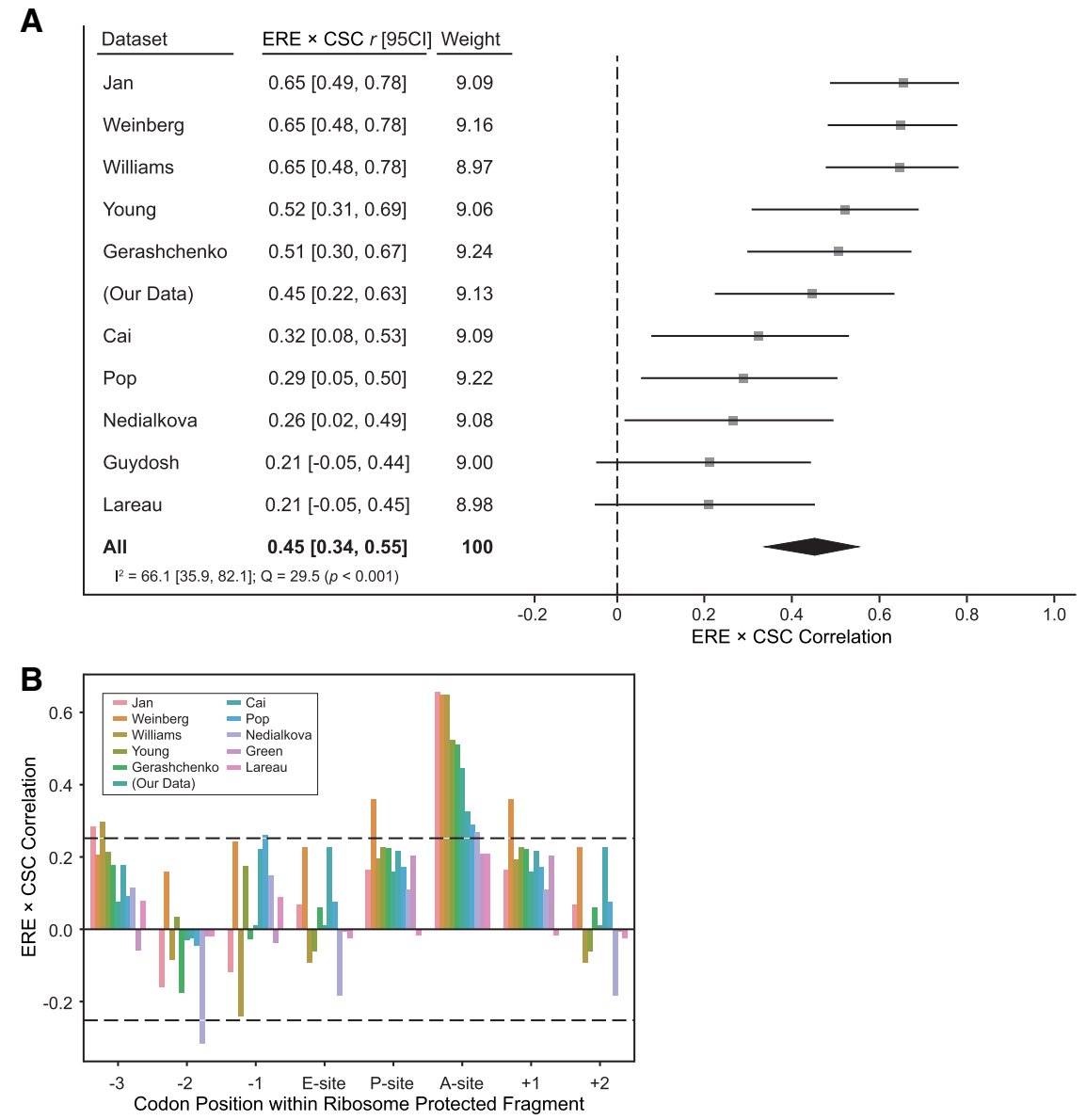

FIGURE 2. Translation elongation rates of codons within the ribosomal A-site correlate with codon influence on mRNA stability. (A) Forest plot describing the meta-analysis of the correlations between normalized EREs and CSCs, a measure of the influence of codons on mRNA stability, using data from 11 cycloheximide-minus ribosome profiling experiments. The correlation between EREs and CSCs for each data set are shown by the squares, with the error bars representing the associated $95 \%$ confidence interval $(95 \mathrm{Cl})$. The combined Pearson correlation estimate is represented by the large black diamond, with the width of the diamond representing the $95 \%$ confidence interval of the aggregate correlation estimate. $I^{2}$ (the percentage of the variation across studies that is attributable to true between-study heterogeneity) and Cochran's $\mathrm{Q}$ are also reported as standard measures of heterogeneity. (B) Bar plot showing the correlation between ERE and CSC values of codons located at specific locations within the RPFs. The E-site, P-site, and A-site correspond to the trinucleotide sequence located at the $+9,+12$, and +15 positions within the RPFs, respectively, and this analysis is extended beyond these positions in either direction. The dashed lines represent the critical $r$ value, such that values outside of the dashed lines represent statistically significant correlations at an uncorrected $\alpha=0.05$.

(Johansson et al. 2011), and certain amino acids are thought to have a significant impact on elongation (Wohlgemuth et al. 2008; Tanner et al. 2009; Watts and Forster 2010; Lareau et al. 2014). However, the observed relationship between estimated tRNA abundance (tAl) and CSC suggests that it is decoding that is driving the relationship between codon identity and mRNA stability rather than other aspects that affect elongation rate (i.e., amino acid identity). To distinguish these possibilities, we plotted the relationship between each codon's ERE and CSC values after grouping the encoded amino acids based on their properties (Fig. 3C). From this analysis, we observe that individual amino acids span a wide range of elongation rates. For example, codons encoding proline and arginine exhibit some of the slowest elongation rates, as one might expect. However, it can readily be observed that for these two amino acids, synonymous codons still span a range of elongation rates that positively correlate with CSC (Fig. 3C). Considering all slopes of the correlation between ERE and CSC for all 18 amino acids decoded by more than one codon, the predominant trend is for these slopes to be positive $\left(t_{(17)}=2.16, P<0.05\right)$, showing that, in general, the faster the elongation rate for a given synonymous codon that encodes a certain amino acid, the higher the associated CSC value. Notably, five amino acids exhibit either no relationship between ERE and CSC for synonymous codons (asparagine) or a negative relationship (phenylalanine, tyrosine, aspartic acid, and histidine). While both tRNA decoding and amino acid identity are thought to influence translation elongation rate, these data suggest that tRNA decoding is primarily driving mRNA decay.

To explore this idea, we reasoned that if both decoding and amino acid contributions to elongation were influencing mRNA decay, then isolating decoding effects on translation elongation should decrease the correlation between transcript-level ERE and mRNA stability. To this end, we corrected the ERE metric calculated for each transcript such that it falls between 0 and 1 , with 0 indicating the use of the slowest elongating codons at every amino acid position, and 1 indicating the use of the fastest elongating codons at every position (see Materials and Methods). This correction for amino acid identity results in the calculation of EREs that only takes into account synonymous codon effects on elongation $\left(E R E_{\text {codons only }}\right)$, in contrast to our original EREs that took into account both codon and amino acid effects on elongation $\left(\mathrm{ERE}_{\mathrm{codons}}+\mathrm{aa}\right)$. Compared to the correlation we previously observed in our data between transcript-level $E R E_{\text {codons + aa }}$ and mRNA HL, the correlation between $\mathrm{ERE}_{\text {codons only }}$ and mRNA HL is significantly higher (Fig. 
A

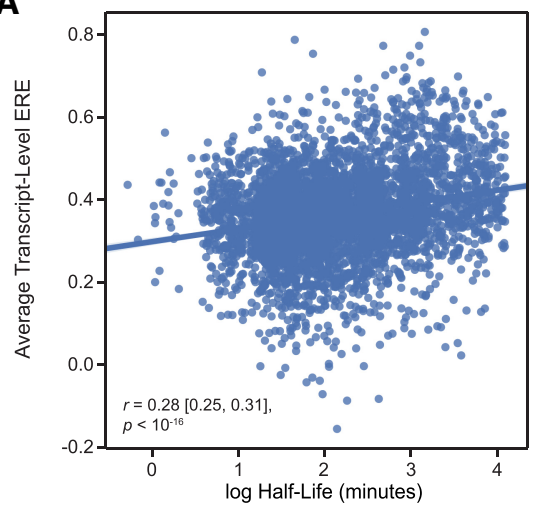

C
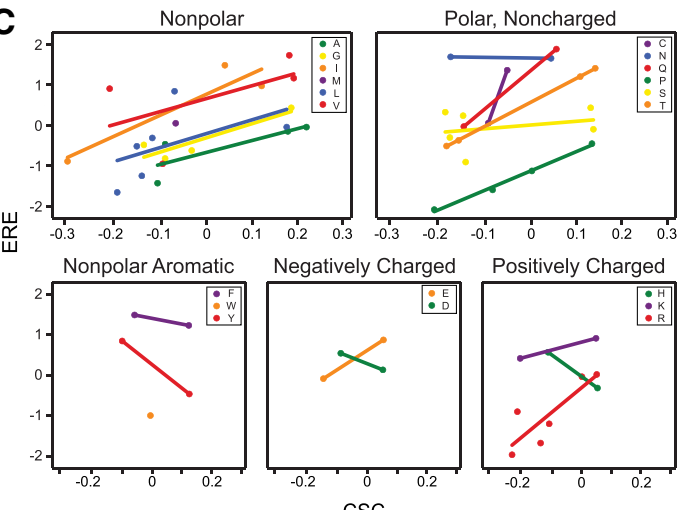

B

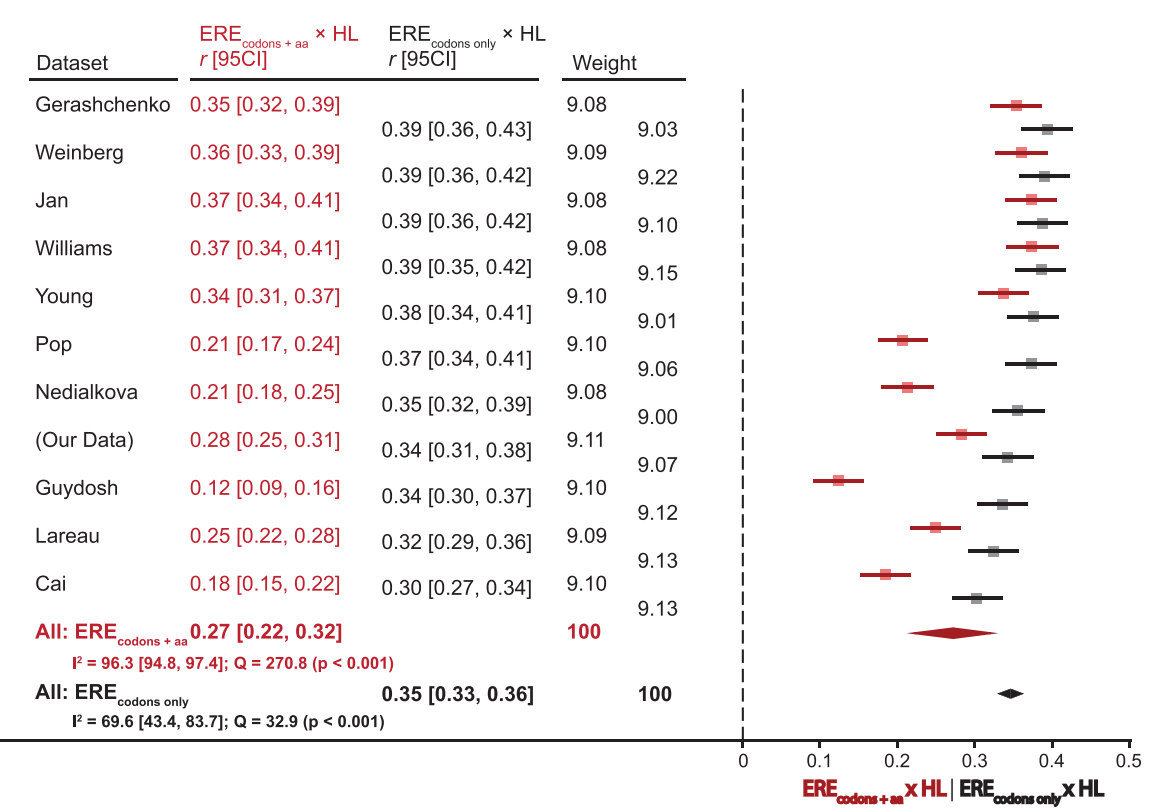

FIGURE 3. Ribosomal A-site decoding drives the relationship between EREs and mRNA stability. (A) Scatter plot and best-fit line describing the relationship between average normalized EREs across a transcript and transcript stability. EREs are calculated using ribosome profiling data from this study, and transcript stability is plotted as the log of the transcript HL (Presnyak et al. 2015). The correlation between transcript-level ERE and transcript $\mathrm{HL}$ takes into account the per-gene variability in these estimates. (B) Forest plot describing the meta-analysis across 11 cycloheximideminus ribosome profiling data sets of the correlation between the log of $\mathrm{mRNA} H \mathrm{HL}$ and either transcript-level ERE (ERE $\mathrm{E}_{\text {codons }+ \text { aai }}$ red squares) or transcript-level ERE that takes into account the influence of codon identity, but not the influence of amino acid identity, on elongation rate

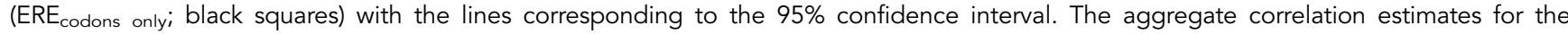
$E R E_{\text {codons + aa }}$ and $E R E_{\text {codons only }}$ analyses are represented by the large red and black diamonds, respectively, with the width of the diamond corresponding to the $95 \%$ confidence interval of the aggregate estimate. $I^{2}$ and Cochran's $Q$ are also reported as standard measures of heterogeneity. (C) Scatter plots of the relationship between normalized per-codon ERE and CSC, with grouping based on the properties of the encoded amino acid. The best-fit lines in each plot describe the relationship between ERE and CSC for each amino acid associated with more than one codon.

$3 B ; r=0.34$ compared to $r=0.28, \Delta r=0.06, N=3969, P<$ 0.01 ), indicating that it is ribosomal A-site decoding that links translation elongation rates to mRNA decay rates.

The true impact of the amino acid correction is evident when we observe the effect of applying this correction to all $S$. cerevisiae data sets considered. While the correlation between $E R E_{\text {codons + aa }}$ and transcript $\mathrm{HL}$ in all data sets analyzed is 0.27 , the correlation becomes even stronger when

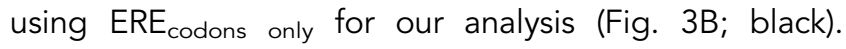
Interestingly, the relationship between $\mathrm{ERE}_{\mathrm{codons}}$ only and transcript HL shows significantly reduced between-data set variability compared to the correlations observed with $E R E_{\text {codons + aa. }}$ This suggests that the primary source of variation between ribosome profiling data sets is in amino acid effects, and not in codon effects within a given amino acid. The clear consensus we observe between the data sets in support of a strong relationship between amino acid-corrected $E R E_{\text {codons only }}$ and $m R N A$ stability is highly consistent with tRNA decoding rate being the primary component of elongation that determines mRNA stability in yeast. 


\section{Ribosomal A-site decoding contributes to translation efficiency}

Our global ribosome profiling analysis indicates that decoding of the codon in the ribosomal A-site, rather than encoded amino acid identity, impacts translation elongation rate and, subsequently, mRNA stability. While these genomic relationships are intriguing, we sought to validate these findings using a controlled system in which synonymous codons are used to manipulate codon optimality while maintaining the encoded amino acid sequence. To this end, we used a set of 11 HIS3 reporters that vary from each other only in their percent codon optimality, from $0 \%-100 \%$ in $10 \%$ increments (Fig. 4A). We have chosen to present these constructs as percent codon optimality for easy comparison with our previous work (Presnyak et al. 2015; Radhakrishnan et al. 2016). While each construct was designed using CSC as the measure of codon optimality (Presnyak et al. 2015; Radhakrishnan et al. 2016), these constructs exhibit a range of average EREs that correlate almost perfectly with codon optimality (Fig. 4A; Supplemental Table S2), an even distribution of ERE (Supplemental Fig. S2A) and optimality (Radhakrishnan et al. 2016) throughout each coding sequence, similar GC content (Supplemental Table S2), and similar minimum free energy (MFE) of predicted secondary structures (Supplemental Table S2; Supplemental Fig. S2B). Importantly, by controlling each of these parameters as well as the encoded His 3 protein sequence and untranslated region sequences in these constructs, we can specifically study the impact of codon decoding on translation efficiency. We previously used these HIS3 reporters to demonstrate the positive correlation between codon optimality and mRNA stability, and we found that even small increases in codon optimality (e.g., 10\%) can cause clearly detectable increases in mRNA stability (Radhakrishnan et al. 2016).

Given the almost perfect correlation between average transcript-level ERE and codon optimality for these HIS3 constructs (Fig. 4A; Supplemental Table S2), we sought to test the effect of codon optimality on translation efficiency while eliminating amino acid and untranslated region-dependent effects. We performed northern blot and western blot analyses to measure the steady state HIS3 mRNA and His3 protein levels expressed from each $0 \%-100 \%$ optimality construct. We observed that whereas the steady state mRNA abundance varied up to approximately ninefold between each of the constructs (Fig. 4B), the steady state protein abundance differences were much more substantial (up to $\sim 49$-fold; Fig. 4 C). Due to the large range in steady state protein abundances that we observed for these constructs, and the relatively small dynamic range of westerns, we present Figure $4 \mathrm{C}$ for illustration purposes but performed protein abundance measurements after diluting samples to variable extents to
A

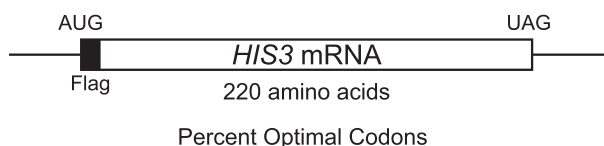

Percent Optimal Codons

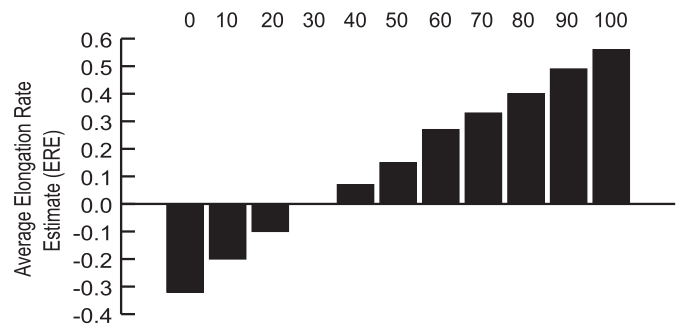

B

Percent Optimal Codons

$\begin{array}{lllllllllll}0 & 10 & 20 & 30 & 40 & 50 & 60 & 70 & 80 & 90 & 100\end{array}$

HIS3 mRNA

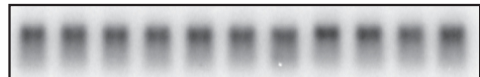

SCR1 RNA

C

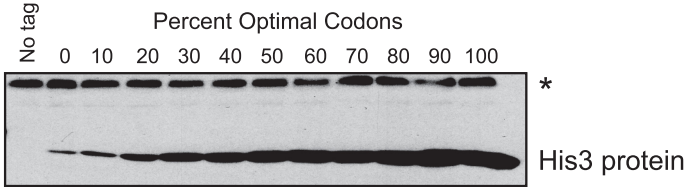

D

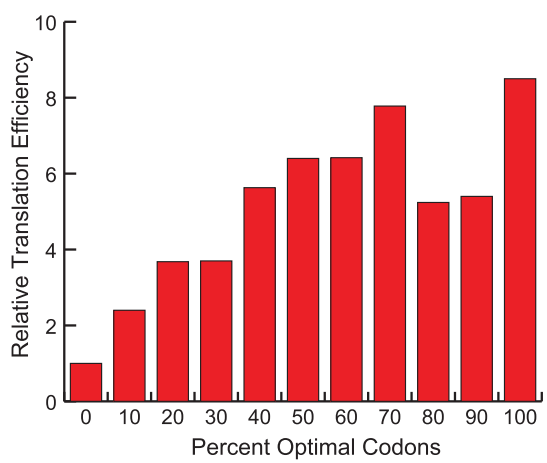

FIGURE 4. Translation efficiency is influenced by codon optimality. (A) Schematic representation of a HIS3 mRNA with an N-terminal FLAG tag. The HIS3 coding sequence was randomly altered using synonymous codons to generate constructs with varying percent codon optimality, from $0 \%$ to $100 \%$. The average ERE for each construct is shown. (B) Steady state HIS3 mRNA levels expressed from the 0\%$100 \%$ optimal HIS3 constructs were analyzed by northern blot and were quantified relative to an SCR1 loading control. (C) Steady state protein levels expressed from the 0\%-100\% optimal HIS3 constructs were analyzed by western blot using anti-FLAG antibody. While the image presented here illustrates the clear correlation between codon optimality and steady state protein abundance using equivalent loading for each sample, more accurate quantification of protein levels was performed using variably diluted samples to minimize protein saturation of the higher optimality constructs. These more accurate values were used for the analysis shown in Figure 4D. The asterisk in this figure indicates the position of a nonspecific band that is indicative of equal loading in all lanes. (D) Graphical representation of the translation efficiency of each HIS3 construct relative to the $0 \%$ optimal construct. Translation efficiencies were determined by dividing the steady state protein abundance by the steady state mRNA abundance for each construct. 
minimize saturation effects. We calculated the protein output per mRNA as a measure of the translation efficiency of each construct and found that as percent codon optimality increases, translation efficiency generally increases (Fig. 4D). Interestingly, similar to our previous observation that even small differences in codon optimality cause detectable differences in mRNA stability, we see that even $10 \%$ differences in codon optimality also result in detectable differences in protein output per mRNA. The positive correlation between codon optimality and both translation efficiency and mRNA stability for this controlled set of constructs suggests that $A$-site decoding rates impact translation efficiency, and these differences in translation efficiency in turn affect the susceptibility of the mRNA to the decay machinery.

\section{Ribosomal A-site decoding contributes to translation kinetics}

As an independent assay to specifically test the influence of codon optimality on translation kinetics, we grew yeast cells expressing each of the $0 \%-100 \%$ optimality HIS3 constructs to mid-log phase before adding ${ }^{35} \mathrm{~S}$-methionine/cysteine and harvesting the cells at different time points. The levels of ${ }^{35} \mathrm{~S}$-methionine/cysteine-labeled His3 protein expressed from each construct at each time point were determined after running immunoprecipitated His3 protein on SDS-PAGE gels and detecting the ${ }^{35} \mathrm{~S}$-labeled protein on a phosphorimager screen. To control for the effects of construct-dependent mRNA abundance or stability differences on the level of ${ }^{35} \mathrm{~S}$-methionine/cysteine-labeled His3 protein produced, we internally normalized the data for each construct by calculating the amount of ${ }^{35} \mathrm{~S}$-labeled His3 protein at each time point relative to the amount detected at the 2 min time point for that construct.

In comparing the two optimality extremes $(0 \%$ and $100 \%$, we observed that the protein expression from the $0 \%$ optimality construct was substantially lower than the protein expression from the $100 \%$ optimality construct, consistent with what we observed in Figure 4C (Fig. 5A). Importantly, the increase in protein expression from one time point to the next was much slower for the $0 \%$ optimality construct relative to the $100 \%$ optimality construct (Fig. $5 A, B)$. Specifically, the rate of ${ }^{35} \mathrm{~S}$ incorporation was 2.8 fold slower for the $0 \%$ optimal construct than for the $100 \%$ optimal construct, indicating that translation of the higher optimality construct is more efficient (Fig. 5C). When analyzing the level of increase in the abundance of ${ }^{35} \mathrm{~S}$-methionine/cysteine-labeled His3 protein over the time course for the remaining nine constructs, we observed a general trend that was consistent with what was observed for the extremes, with relative ${ }^{35} \mathrm{~S}$ incorporation rates correlating with codon optimality (Fig. 5B,C). These data are in agreement with our previous observations

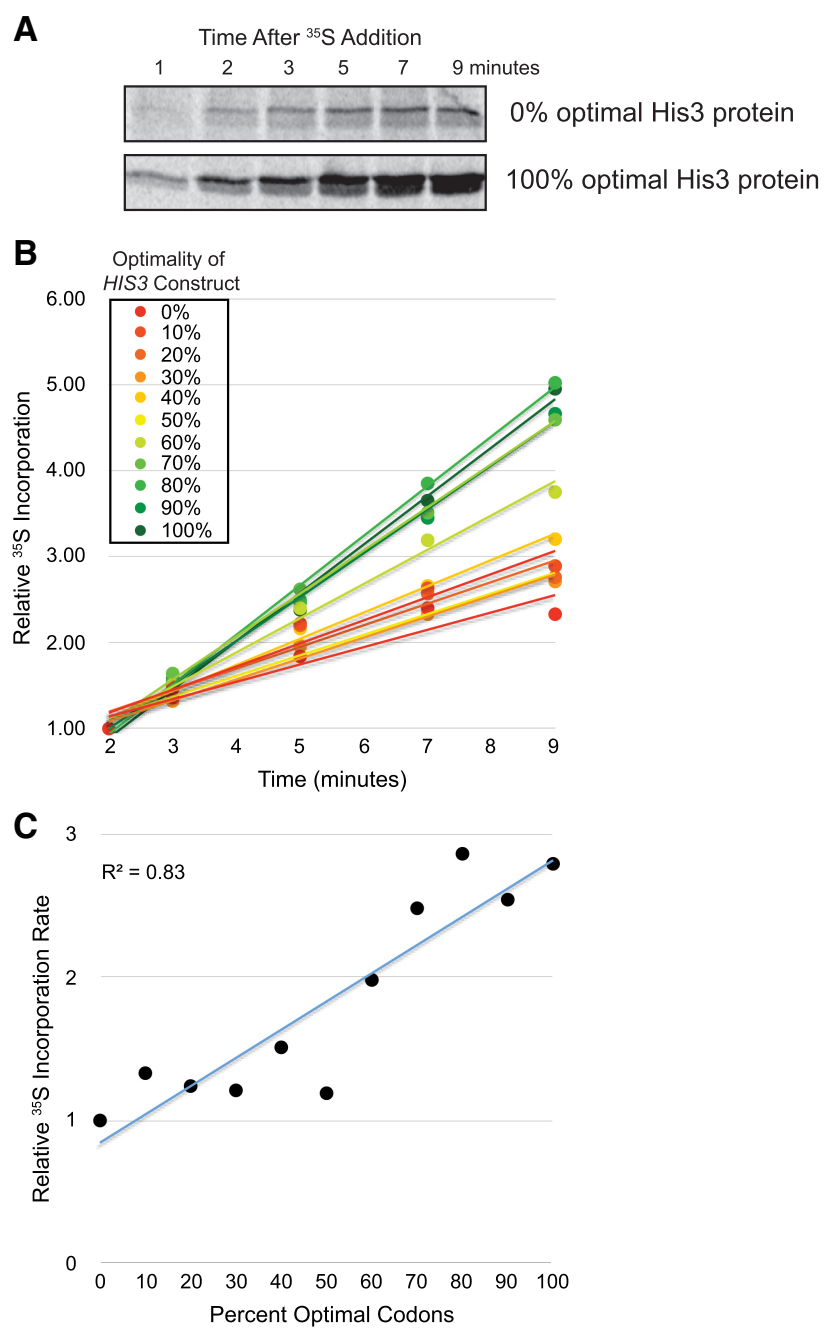

FIGURE 5. Codon optimality contributes to the rate of His3 protein production during ${ }^{35} \mathrm{~S}$-methionine/cysteine labeling. (A) A representative image showing levels of ${ }^{35} \mathrm{~S}$-methionine/cysteine-labeled His3 protein at each time point following addition of ${ }^{35} \mathrm{~S}$-methionine/cysteine to $S$. cerevisiae cells at mid-log phase. His3 protein expressed from the $0 \%$ and $100 \%$ optimal HIS3 constructs is presented. (B) Graphical representation of the increase in ${ }^{35} \mathrm{~S}$-methionine/cysteine-labeled His3 protein abundance over time, relative to the protein abundance at the 2 min time point, for each of the $0 \%-100 \%$ optimal HIS3 constructs. (C) The slope of each curve in Figure 5B, normalized to the slope for the $0 \%$ optimal construct, is plotted as a measure of the relative rate of ${ }^{35} \mathrm{~S}$ incorporation per minute for each HIS3 construct.

and suggest that A-site decoding is linking translation kinetics to mRNA stability.

\section{DISCUSSION}

Previously, we had established that codon optimality is a powerful determinant of mRNA decay rates in the yeast S. cerevisiae (Presnyak et al. 2015). Other labs subsequently established this as a general principle across a number of species (Bazzini et al. 2016; Boël et al. 2016; 
Harigaya and Parker 2016; Mishima and Tomari 2016), leading to widespread interest in the general rules that dictate how translation elongation rates are communicated to the decay machinery. Our previous studies using reporter constructs suggest that decoding of codons in the A-site of the ribosome dictate elongation rate, which in turn sets mRNA decay rate via recruitment of the decapping factor Dhh1 (Radhakrishnan et al. 2016). However, whether codon optimality-mediated differences in translation elongation link directly to differences in mRNA stability on a genome-wide scale has not been previously shown.

We first performed ribosome profiling without cycloheximide in yeast as was described previously (Hussmann et al. 2015; Weinberg et al. 2016) and were able to extract estimates of codon-specific elongation rates that correlate well with the tRNA adaptation index (tAl). Of note, these findings are in agreement with a number of ribosome profiling studies lacking cycloheximide (Hussmann et al. 2015; Weinberg et al. 2016). Further, codon-specific EREs correlate with codon occurrence to mRNA stability correlations (CSCs) that we calculated previously (Presnyak et al. 2015), suggesting that elongation rate at $\mathrm{A}$-site codons genomewide is influencing decay (Fig. 1C). It is important to note that while decay measurements were taken from a different strain (rpb1-1) at a different temperature, these mRNA half-lives correlate well with half-lives determined by thiolutin shut-off, metabolic labeling, or shut-off of reporter mRNA using inducible promoters in wildtype cells under standard conditions (Herrick et al. 1990; Presnyak et al. 2015; Radhakrishnan et al. 2016).

Using our calculated per-codon ERE, we sought to determine how transcript-level elongation rates might influence decay. As would be predicted from codon-level elongation rates, transcripts with increasing proportions of optimal codons exhibit higher average EREs (Fig. 1D). Extending these data revealed that transcript-level EREs positively correlate with mRNA half-lives (Fig. 3A). Further analysis revealed that 10 previously published ribosome profiling data sets also yield a significant ERE by mRNA $\mathrm{HL}$ correlation (Fig. 3B).

The correlations we observed between ERE and mRNA $\mathrm{HL}$ in the 11 ribosome profiling data sets used in our study are statistically significant, but represent an aggregated correlation coefficient of only 0.27 . Correlation of ERE at particular transcript positions with mRNA HL reveals no clear position-dependent effects of codons (Supplemental Fig. S3). While these data sets are all derived from similar strains grown under similar conditions (Supplemental Table S1), the aggregated correlation coefficient is lowered by the presence of four data sets that show weaker correlation with all other data sets (Supplemental Fig. S1). While Figure $3 \mathrm{~B}$ suggests that amino acid effects contribute to much of the variability between data sets, we do not know the precise cause of this variation. Thus, we included all data sets to provide a conservative estimate.
In addition to technical considerations, there are other reasons for why a high correlation coefficient between ERE and mRNA HL would not be expected. For example, there are likely complex interactions between codons within open reading frames that are not accounted for by our modeling such as codon context (Chevance et al. 2014; Gamble et al. 2016), synonymous codon co-occurrence bias (Quax et al. 2015), and codon triplet effects (Chevance and Hughes 2017). In addition, mRNA decay is not solely influenced by translation elongation, with clear contributions from other steps in translation and other mRNA features such as open reading frame length and cis-acting sequences in untranslated regions (Muhlrad and Parker 1992; Lee and Lykke-Andersen 2013; Geisberg et al. 2014; Neymotin et al. 2016; Cheng et al. 2017). Together, these data extend previously observed correlations between codon-specific tAl and elongation rate by demonstrating that a statistically significant global relationship exists between transcript-level EREs and mRNA stability.

Translation elongation is a combination of decoding at the A-site as well as peptide bond formation rates that differ among amino acids (Watts and Forster 2010; Lareau et al. 2014). To uncover mechanistic features of how elongation might influence decay, we showed that EREs of synonymous codons generally positively correlate with the impact of codons on mRNA stability, independent of the encoded amino acid (Fig. 3C). Further, when we isolate synonymous codon effects on elongation from amino acid-specific effects, our correlations with mRNA HL are improved (Fig. 3B). If amino acid-specific effects on elongation were contributing to mRNA decay rates, we would have seen a weakened correlation between codon-specific elongation rate ( $\left.\mathrm{ERE}_{\text {codons only }}\right)$ and $\mathrm{CSC}$ (Fig. 3B). In addition, if amino acid identity substantially impacted mRNA decay rates, we would not have expected that most amino acids are encoded by synonymous codons that both contribute to the stabilization (positive CSC) and destabilization (negative CSC) of mRNAs. This highlights that A-site tRNA decoding is likely driving mRNA decay in yeast.

To rigorously test whether A-site decoding is affecting translation elongation which in turn affects decay, we utilized a set of 11 HIS3 constructs that differ in total codon optimality from $0 \%-100 \%$ in $10 \%$ increments. These constructs have the same untranslated regions, the same initiation context, and encode the exact same protein, making them an ideal system to isolate the effects of A-site decoding and to test incremental increase in the proportion of optimal codons. We had previously shown that the mRNAs expressed from these constructs exhibit a $\sim 13-$ fold range in half-lives, with a $\sim 3 \mathrm{~min} \mathrm{HL}$ for the $0 \%$ optimal construct and a 40 min HL for the 100\% optimal construct (Radhakrishnan et al. 2016). In the present study, we find a roughly 8.5-fold range of translation efficiency (protein per mRNA; Fig. 4) and a 2.8 -fold range of relative ${ }^{35} \mathrm{~S}$-methionine/cysteine incorporation rate across the HIS3 constructs 
(Fig. 5), indicating differences in translation kinetics that are driven by different rates of codon decoding. These data are in agreement with our previous observation that upon inhibiting translation initiation through glucose deprivation, existing ribosomes on the 100\% optimal HIS3 construct were cleared from the mRNA faster than ribosomes associated with the $0 \%$ optimal HIS3 construct, presumably through more rapid elongation (Presnyak et al. 2015). While the codon-mediated effects on kinetics that we observed for this set of HIS3 constructs are weaker than the range of translation efficiencies or half-lives observed, all measures trend in the same direction indicating that codon optimality drives changes in translation kinetics that in turn drive changes in mRNA decay rates.

This work makes two significant advances toward understanding the intimate relationship between mRNA translation and decay. First, we show that codon and transcriptlevel EREs correlate with mRNA stability across the transcriptome. Second, we identify ribosomal A-site decoding as the step of elongation that impacts normal mRNA decay in yeast. In contrast, other work has suggested that both codon optimality and amino acid identity are important for influencing mRNA stability in zebrafish and Xenopus (Bazzini et al. 2016). These differences between yeast and higher eukaryotes may reflect differences in either the translation machinery, the decay machinery, or both. Alternatively, amino acid effects could be explained by the influence of amino acids on mRNA decay independent of translation elongation. For example, one possibility is that particular nascent amino acid sequences could recruit trans-acting factors that in turn regulate mRNA stability.

Strikingly, these findings are consistent with other known decay pathways: no-go, nonstop, and nonsensemediated mRNA decay all are triggered by different states of the ribosomal A-site. In future work, it will be fascinating to determine how A-site decoding rates are transmitted to the normal mRNA decay machinery in yeast and also to determine how translation elongation influences decay in higher eukaryotes.

\section{MATERIALS AND METHODS}

\section{Yeast strains}

The genotypes of the $S$. cerevisiae strains used in this study are listed in Supplemental Table S3. Yeast cells were grown to midlog phase at $24^{\circ} \mathrm{C}$ in synthetic media ( $\mathrm{pH} 6.5$ ) containing $2 \%$ glucose and appropriate amino acids.

\section{Ribosome profiling library preparation}

Using S. cerevisiae strain yJC2229, ribosome footprint RNA and control total RNA were isolated and libraries were prepared as was previously described (Smith et al. 2014), with slight modifications. Specifically, cycloheximide treatment was omitted prior to cell harvest but was included during cell lysis. RNA purified from monosome fractions and control total RNA were depleted of ribosomal RNA once using the Yeast Ribo-Zero Gold rRNA Removal Kit (Illumina MRZY1324) according to the manufacturer's instructions following the addition of an RNA Spike-In mix (Thermo Fisher Scientific 4456740) to the total RNA sample. cDNA libraries were amplified using the indexed primer oKB690 (Smith et al. 2014) and were sequenced at the Case Western Reserve University Genomics Core Facility using the Illumina HiSeq2500 platform.

\section{Ribosome profiling and RNA sequencing data processing}

Adaptors were trimmed from the RNA and ribosome profiling data sets using cutadapt with the following parameters: -a CTGTAGGCACCATCAAT -trim-n -m 24 -M 36 -0 6. Other data sets were subject to adaptor trimming as appropriate using study-specific adaptor sequences, but otherwise identical trimming parameters. The ribosome profiling reads were then aligned against an index of $S$. cerevisiae ribosomal RNA sequences from Ensembl using bowtie with the following parameters: -D $15-R 2$ $N 1-L 25-I S, 1,0.75$. Sequences that failed to align to the ribosomal RNA index were taken to be from messenger RNA and were aligned to the entire $S$. cerevisiae genome using HISAT2 with release 84 of Ensembl's gene annotations of the sacCer3 genome (ftp://ftp.ensembl.org/pub/release-84/gtf/saccharomyces_cerevisiae/Saccharomyces_cerevisiae.R64-1-1.84.gtf.gz) to guide alignment to the transcriptome. Finally, multi-mapped reads were discarded and uniquely mapped ribosome footprint reads were transformed to transcriptome-based coordinates for further analysis using the sam2transcriptome python script. Ribosome footprint reads were assigned to the A-site codon using the method outlined previously (Ingolia et al. 2009), where the P-site is identified based on the well-characterized pileup of RPFs over the start codon, with the P-site generally located 12 nt into the fragments located at this start site peak, and the Asite another $3 \mathrm{nt}$ past this point. RNA-sequencing data was quantified with htseq-count (Anders et al. 2015) to estimate per-gene expression as reads per million mapped reads (RPM). For any ribosome profiling data sets without RNA-seq data, we used per-gene fragments per kilobase of transcript per million mapped reads (FPKMs) calculated as the mean of RNA-seq data from all comparable data sets.

\section{Model}

Our primary aim was to extract estimates of the relative rates at which ribosomes move off of specific codons. Per-codon elongation rates have been estimated in the past (Qian et al. 2012; Pop et al. 2014; Hussmann et al. 2015; Weinberg et al. 2016). However, the common approach to estimating codon-specific elongation times has been to simply calculate the ribosome density over a codon relative to the frequency of a codon in an mRNA, and then sum these values up across the entire transcriptome. This approach is intuitive and easily calculated, but it is difficult to assess the confidence of the estimates generated from this procedure. There is often no control for biases introduced by those 
genes with sparse RPF coverage, which are weighted equally with the rest of the transcriptome in the default approach.

To extract more robust estimates of codon-specific ribosome elongation rates, we propose the following model, which is based on the assumption that ribosome initiation is typically a much slower process than elongation (Shah et al. 2013). Let $\rho_{g}$ represent the initiation rate for a gene $g$, and $\tau_{c}$ be the average elongation time for a codon type $c \in\{A A A, A A C, A A G, \ldots, T T T\}$. Thus, under our assumption, for a single mRNA molecule from gene $g$, the proportion of time that a ribosome can be found on a position with codon $c$ is:

$$
\rho_{g} \tau_{c}
$$

Let the relative concentration, as measured by RNA-seq reads mapped to gene $g$ per million mapped reads for gene $g$ be denoted by $M_{g}$, and let the number of $c$ codons in gene $g$ be $N_{g, c}$. Also, let $Y_{g, c}$ be the number of ribosome footprints mapping to $c$ codons in gene $g$. Based on this, we assume that $Y_{g, c}$ follows an overdispersed Poisson distribution with the following mean:

$$
\begin{aligned}
E\left(Y_{g, c}\right) & =M_{g} N_{g, c} \rho_{g} \tau_{c} \\
& =\exp \left[\log \left(M_{g} N_{g, c}\right)+\log \left(\rho_{g}\right)+\log \left(\tau_{c}\right)\right] .
\end{aligned}
$$

An overdispersed Poisson distribution was chosen to model ribosomal reads as it has previously been found that the sampling rate of RNA fragments from their associated transcripts can rarely be described as following a Poisson distribution due to uneven scaling of variability with the mean (Soneson and Delorenzi 2013). It must be understood that there is a degeneracy in this model; there are an infinite number of parameters which fit the data equally well. However, we can estimate the values $\tau_{c}$ relative to $\rho_{g}$. To estimate the model parameters, we used a generalized linear mixed model which assumes that $\log \left(\rho_{g}\right) \sim \operatorname{Normal}\left(0, s^{2}\right)$ where $s^{2}$ and $\log \left(\tau_{c}\right)$ are all estimated from the data using maximum likelihood with an overdispersed Poisson distribution. The mixed model gets around the degeneracy (i.e., identifiability problem) by assuming that on average across all genes $\mathrm{E}\left[\log \left(\rho_{g}\right)\right]=0$. However, it must be understood that the resultant parameter estimates $\left(\hat{\rho}_{g}\right.$ and $\left.\hat{\tau}_{c}\right)$ are relative. Given that estimates of codon-specific elongation times are relative, we will adopt the convention of first calculating the relative elongation rate $\hat{\lambda}_{c}=\hat{\rho}_{c}{ }^{-1}$ and then normalizing the resultant rate estimates so that $\mathrm{E}\left[\hat{\lambda}_{c}\right]=0$ and $s^{2}=1$, for ease of interpretability.

This model implicitly more highly weights those transcripts with a greater density of information on ribosome dynamics, removing potential biases due to sparse RPF density and avoiding the need to set an arbitrary threshold for ribosome coverage in an attempt to mitigate these effects. However, because the model can be easily represented as a linear mixed effects model, it can be estimated directly from ribosome profiling data using out of the box solvers in MATLAB or R, making it readily applicable to a variety of data sets.

\section{Ribosome profiling and RNA sequencing data analysis}

For each gene, we first found the number of codons $c\left(N_{g, c}\right)$ in gene $g$, and also calculated the total number of RPFs over gene $g$ with the A-site mapped to codon $c\left(Y_{g, c}\right)$. Relative RNA concen- tration $\left(M_{g}\right)$ was calculated using htseq-count and standardized as RPM, as outlined above. This procedure was performed for each gene in the data set, with no restriction on total ribosome density or gene length, though only genes with nonzero RNA expression and RPF counts are included. Data from between 4188 and 5245 genes were included, depending on the data set analyzed, ensuring that we were able to leverage the majority of protein coding genes for analysis. For the model, data were structured as longform arrays, with the number of rows equal to the product of the number of genes considered times the number of amino acid-coding codons (61), and the number of columns equal to 5 (codon identity, gene identity, number of RPFs over a given codon in a gene, the total occurrence of that codon in a gene, and the estimate of gene expression). The model itself was fit using the "fitglme" function in the Statistical Toolbox of MATLAB, version R2016a. The code used to form ribosome profiling data into appropriate data sets, and the MATLAB code used to specify and run the model is available as Supplemental Code. All other statistical analyses were carried out using the statsmodels and scipy packages for Python, and Matplotlib and Seaborn were used to generate Figures 1-3. The method used to calculate the statistical significance of a change in Pearson correlation coefficient was taken from (Cohen et al. 2013). Briefly, the test statistic for the change in the Pearson correlation coefficient can be found as follows:

$$
z_{1-2}=\frac{\tanh ^{-1}\left(r_{1}\right)-\tanh ^{-1}\left(r_{2}\right)}{\sqrt{\left(N_{1}-3\right)^{-1}+\left(N_{2}-3\right)^{-1}}},
$$

where $N_{i}$ is the number of observations used to calculate $r_{i}$. This statistic is drawn from a normal distribution, and the corresponding $P$-value can be found with the survival function of a normal distribution with $\mu=0$ and $\sigma=1$.

\section{Calculation of robust Pearson correlation coefficients}

The standard method for calculating Pearson correlation coefficients has no facility to take into account the precision associated with the individual quantities that make up vectors $\mathbf{X}$ and $\mathbf{Y}$, the two vectors for which the correlation is to be calculated. Therefore, we chose to leverage the modeling capabilities of Stan (Carpenter et al. 2017) to specify a model where $\mathbf{X}$ and $\mathbf{Y}$ are latent variables sampled from a Gaussian distribution such that $\mathbf{X} \sim \operatorname{Normal}\left(\overline{\mathbf{X}}, \Lambda_{1}\right)$ and $\mathbf{Y} \sim \operatorname{Normal}\left(\overline{\mathbf{Y}}, \Lambda_{2}\right)$, where $\overline{\mathbf{X}}$ and $\overline{\mathbf{Y}}$ are the point estimates for each quantity, and $\Lambda_{1}$ and $\Lambda_{2}$ are the standard errors associated with the values in $\overline{\mathbf{X}}$ and $\overline{\mathbf{Y}}$, respectively. $\overline{\mathbf{X}}$ and $\overline{\mathbf{Y}}$ may be CSC values and ribosome EREs, respectively, or mRNA half-lives and transcript-level average elongation rates, depending on the needs of the analysis. Finally, we find the value of $r$, the Pearson correlation coefficient, to maximize the likelihood of the latent variables $\mathbf{X}$ and $\mathbf{Y}$ as samples drawn from a multivariate Gaussian distribution with means $\mu_{X_{1}} \mu_{Y}$ and the covariance matrix described as follows:

$$
(\mathbf{X}, \mathbf{Y}) \sim \operatorname{MvGaussian}\left(\left(\mu_{X}, \mu_{Y}\right),\left[\begin{array}{cc}
\sigma_{1}^{2} & \sigma_{1} \sigma_{2} r \\
\sigma_{1} \sigma_{2} r & \sigma_{2}^{2}
\end{array}\right]\right),
$$

where $\sigma_{1}$ and $\sigma_{2}$ are the standard deviations of $\mathbf{X}$ and $\mathbf{Y}$, respectively, and are also estimated in the model. The resultant sampled $r$ values then represent the maximum a posteriori (MAP) distribution of $r$. The code to accomplish this was adapted from previous 
work (Lee and Wagenmakers 2014; Schwarzer et al. 2015). All models in Stan were run with 1000 warmup samples and 1000 acquisition samples, across four separate chains. Models were assessed for convergence by ensuring that the scale reduction factor across chains, was equal to 1 .

\section{Meta-analysis}

The meta-analysis of Pearson correlation coefficients follows the Inverse Variance method for calculating the random effects estimate $\theta_{R}$, using the DerSimonian-Laird estimator for calculating the between-study variability parameter $\tau^{2}$, and using the Hartung and Knapp correction when estimating $\operatorname{var}\left(\theta_{R}\right)$. All meta-analyses were implemented in Python using custom code based on the previously described methods (Schwarzer et al. 2015). Briefly, let the population estimate of the correlation between CSC and translation elongation rate (or mRNA HL and average elongation rate), $r$, be expressed as the normally distributed Fisher's $z=\tanh ^{-1}(r)$, with the point estimate and variance of $z$ estimated from the a posteriori distribution of $r$ calculated from Stan. With $z$ sampled from a normal distribution with known variance, for each data set we can define individual estimates of $z$ for data set $k$ as:

$$
z_{k}=\bar{z}+u_{k}+\sigma_{k} \in_{k},
$$

where $\bar{z}$ is the true transformed correlation coefficient in the population of data sets, $u_{k} \sim N\left(0, \tau^{2}\right)$ captures the error due to heterogeneity between studies, while $\varepsilon_{k} \sim N(0,1)$, scaled by the known standard error of $\sigma_{k}^{2}=\operatorname{var}\left(z_{k}\right)$, represents within-study error. The estimate of $\bar{z}$ is simply the weighted average of individual estimates $z_{k}$, where the weight with which each study is considered in the analysis, $w_{k}^{*}$, is inversely proportionate to the sum of the study-specific variance plus the between-study variance. $w_{k}^{*}=1 /\left(\tau^{2}+\sigma_{k}^{2}\right)$, leaving us with

$$
\begin{gathered}
\bar{z}=\frac{\sum_{k=1}^{K} w k * z k}{\sum_{k=1}^{K} w k *}, \\
\operatorname{var}(\bar{z})=\frac{1}{K} \sum_{k=1}^{K} \frac{w_{k}^{*}}{\sum_{k=1}^{K} w k *}\left(z_{k}-\bar{z}\right)^{2} .
\end{gathered}
$$

In order to calculate the $95 \%$ confidence interval around $\bar{z}$, take $\bar{z} \pm t_{\left(k-1 ; 1-\frac{\alpha}{2}\right)} \sqrt{\operatorname{var}(\bar{z})}$. To express the results of this analysis is terms of $\bar{r}$, simply take the hyperbolic tangent of $\bar{z}$ and the upper and lower bounds of $\bar{z}$ calculated above.

\section{Calculation of amino acid-corrected elongation rate}

An imaginary polypeptide can be perfectly efficient if every amino acid is coded with the fastest elongating codon, or perfectly inefficient if every amino acid is coded with the slowest elongating codon. Any further changes would require altering the amino acid sequence. We wished to calculate the elongation rate of a natural transcript relative to these two extremes, in order to remove effects of amino acid choice and effectively correct for any biases due to a transcript being enriched in amino acids that happen to be associated with faster or slower total ribosome transit times, regardless of the decoding speed. To accomplish this, we use EREs to find the maximum and minimum elongation rate associated with each amino acid. Then, for a given transcript, we calcu- late the hypothetical maximum $\left(\overline{E R}_{\text {max }}\right)$ and minimum $\left(\overline{E R}_{\text {min }}\right)$ average elongation rates given the amino acid sequence associated with the transcript. The correction is then calculated as follows:

$$
\text { ERE codons only }=\frac{\left(\overline{E R}_{\text {actual }}-\overline{E R}_{\text {min }}\right)}{\left(\overline{E R_{\text {max }}}-\overline{E R}_{\text {min }}\right)},
$$

where $\overline{E R}_{\text {actual }}$ is the average elongation rate based on the actual codon sequence of the transcript.

\section{Northern and western blot analysis}

S. cerevisiae cells expressing N-terminally FLAG-tagged $0 \%-$ $100 \%$ optimal HIS3 (yJC2419 to yJC2429), or an untagged $100 \%$ optimal HIS3 control (yJC2088), were used for total RNA or protein isolation as previously described (Geisler et al. 2012). Equal amounts of each RNA were run on 1.4\% agarose formaldehyde gels, transferred onto nylon membrane, and probed using a ${ }^{32} \mathrm{P}$ end-labeled oligonucleotide (oJC2564) (Presnyak et al. 2015), which is complementary to a $23 \mathrm{nt}$ region within each HIS3 construct that was maintained for detection purposes. For protein analysis, either an equal amount of each sample, or variable amounts of each sample (in order to enable more accurate protein abundance quantification by minimizing signal saturation), were run on SDS-PAGE gels and were transferred onto PVDF membrane. His3 protein was detected using rabbit anti-FLAG primary antibody (Sigma F7425) and goat anti-rabbit secondary antibody (Pierce 31460). Steady state RNA and protein abundance was quantified using ImageQuant and ImageJ software, respectively. Translation efficiency was determined by dividing the steady state His3 protein abundance by the steady state HIS3 mRNA abundance for each construct.

\section{${ }^{35}$ S-methionine/cysteine incorporation and FLAG- His3 protein immunoprecipitation assay}

S. cerevisiae cells expressing N-terminally FLAG-tagged 0\%$100 \%$ optimal HIS3 (yJC2488 to yJC2498), were grown in the absence of supplemented methionine and cysteine. At mid-log phase, the cells were concentrated 10 -fold by pelleting and resuspending in the same media used for growth. Next, $15 \mu \mathrm{L}$ of ${ }^{35} \mathrm{~S}$ methionine/cysteine mix (PerkinElmer NEG072) per $50 \mathrm{~mL}$ of cells were added to each culture and cell aliquots were transferred into an equal volume of $2 \times$ Buffer A ( $50 \mathrm{mM}$ sodium azide, $100 \mathrm{mM}$ $\mathrm{NaCl}, 10 \mathrm{mM}$ Tris- $\mathrm{HCl} \mathrm{pH} 7.4,5 \mathrm{mM} \mathrm{MgCl}, 5 \mathrm{mM} \mathrm{NH}_{4} \mathrm{Cl}, 1$ $\mathrm{mM}$ DTT, $2 \mu \mathrm{L} / \mathrm{mL}$ protease inhibitor [Sigma p8215], and 200 $\mu \mathrm{g} / \mathrm{mL}$ cycloheximide) on ice at the time points indicated in Figure 5A. Cells were pelleted, washed, and then lysed in $1 \times$ Buffer A using glass beads and alternating cycles of vortexing and incubating on ice. Lysates were quantified and an equivalent amount of lysate for each time point within a time course (typically between 25 and $40 \mathrm{OD}_{260}$ units) were incubated with rabbit antiFLAG antibody $(13.3 \mu \mathrm{L} / \mathrm{mL})$ in $1 \times$ Buffer A supplemented with $\mathrm{NP} 40$ to $0.1 \%$ and $3 \mu \mathrm{L} / \mathrm{mL}$ protease inhibitor for $1 \mathrm{~h} 10 \mathrm{~min}$. at $4^{\circ} \mathrm{C}$. Anti-FLAG antibody-bound His3 protein was then immunoprecipitated following a $1 \mathrm{~h}$ incubation with Dynabeads Protein G (Invitrogen 10004D). After removal of the supernatant, the Dynabeads were washed 5 times with IP Wash Buffer $(150 \mathrm{mM}$ 
Tris- $\mathrm{HCl} \mathrm{pH} \mathrm{7.5,} 125 \mathrm{mM} \mathrm{NaCl}, 2 \mathrm{mM} \mathrm{MgCl}_{2}$, and 0.1\% NP40) and FLAG-His3 protein was eluted by heating the beads at $95^{\circ} \mathrm{C}$ for 5 min. in IP Elution Buffer $(50 \mathrm{mM}$ Tris- $\mathrm{HCl} \mathrm{pH} \mathrm{7.5,0.5 \%} \mathrm{SDS,}$ and $50 \mathrm{mM}$ EDTA pH 8.0). Eluates were run on SDS-PAGE gels, transferred onto PVDF membrane, and then exposed to a phosphorimager screen. Detected ${ }^{35} \mathrm{~S}$-labeled His3 protein was quantified using ImageQuant software and normalized to the 2 min time point for each time course.

\section{DATA DEPOSITION}

The data sets generated and/or analyzed during the current study are available in the Gene Expression Omnibus. Details and GEO numbers for each data set are in Supplemental Table S1.

\section{SUPPLEMENTAL MATERIAL}

Supplemental material is available for this article.

\section{ACKNOWLEDGMENTS}

We thank all members of the Coller laboratory and the laboratory of Dr. Kristian Baker for helpful comments and discussion. We also thank DaJuan Whiteside for technical assistance. This work was supported by National Institutes of Health grant GM080464 to J.C., National Institutes of Health T32 grant GM007250 for G. $\mathrm{H}$., and National Institutes of Health grants R01GM118018 and R01GM125086.

Author contributions: G.H. and N.M. developed the statistical models detailed in the Methods section for analyzing ribosome profiling data. N.A. performed experiments for Figures 1-5. G.H. performed the analyses for Figures 1-3. G.H., N.A., T.S., and J.C. wrote the manuscript and all authors read and approved the final manuscript.

Received April 12, 2018; accepted July 6, 2018.

\section{REFERENCES}

Anders S, Pyl PT, Huber W. 2015. HTSeq-a Python framework to work with high-throughput sequencing data. Bioinformatics 31: 166-169.

Bazzini AA, Del Viso F, Moreno-Mateos MA, Johnstone TG, Vejnar CE, Qin Y, Yao J, Khokha MK, Giraldez AJ. 2016. Codon identity regulates mRNA stability and translation efficiency during the maternal-to-zygotic transition. EMBO J 35: 2087-2103.

Boël G, Letso R, Neely $H$, Price WN, Wong K-H, Su M, Luff JD, Valecha M, Everett JK, Acton TB, et al. 2016. Codon influence on protein expression in $E$. coli correlates with mRNA levels. Nature 529: 358-363.

Cai Y, Futcher B. 2013. Effects of the yeast RNA-binding protein Whi3 on the half-life and abundance of CLN3 mRNA and other targets. PLoS One 8: e84630.

Carpenter B, Gelman A, Hoffman MD, Lee D, Goodrich B, Betancourt M, Brubaker MA, Guo J, Li P, Riddell A. 2017. Stan: a probabilistic programming language. J Stat Softw 76: 1-32.

Cheng J, Maier KC, Avsec Z, Rus P, Gagneur J. 2017. Cis-regulatory elements explain most of the mRNA stability variation across genes in yeast. RNA 23: 1648-1659.
Chevance FFV, Hughes KT. 2017. Case for the genetic code as a triplet of triplets. Proc Natl Acad Sci 114: 4745-4750.

Chevance FF, Le Guyon S, Hughes KT. 2014. The effects of codon context on in vivo translation speed. PLoS Genet 10: e1004392.

Cohen J, Cohen P, West SG, Aiken LS. 2013. Applied multiple regression/correlation analysis for the behavioral science. Routledge, NY.

Coller J, Parker R. 2004. Eukaryotic mRNA decapping. Annu Rev Biochem 73: 861-890.

dos Reis M, Savva R, Wernisch L. 2004. Solving the riddle of codon usage preferences: a test for translational selection. Nucleic Acids Res 32: 5036-5044.

Gamble CE, Brule CE, Dean KM, Fields S, Grayhack EJ. 2016. Adjacent codons act in concert to modulate translation efficiency in yeast. Cell 166: 679-690.

Geisberg JV, Moqtaderi Z, Fan X, Ozsolak F, Struhl K. 2014. Global analysis of mRNA isoform half-lives reveals stabilizing and destabilizing elements in yeast. Cell 156: 812-824.

Geisler S, Lojek L, Khalil AM, Baker KE, Coller J. 2012. Decapping of long noncoding RNAs regulates inducible genes. Mol Cell 45: 279-291.

Gerashchenko MV, Gladyshev VN. 2014. Translation inhibitors cause abnormalities in ribosome profiling experiments. Nucleic Acids Res 42: e134.

Ghosh S, Jacobson A. 2010. RNA decay modulates gene expression and controls its fidelity. Wiley Interdiscip Rev RNA 1: 351-361.

Guydosh NR, Green R. 2014. Dom34 rescues ribosomes in 3' untranslated regions. Cell 156: 950-962.

Harigaya Y, Parker R. 2016. Analysis of the association between codon optimality and mRNA stability in Schizosaccharomyces pombe. BMC Genomics 17: 895

Herrick D, Parker R, Jacobson A. 1990. Identification and comparison of stable and unstable mRNAs in Saccharomyces cerevisiae. Mol Cell Biol 10: 2269-2284.

Hussmann JA, Patchett S, Johnson A, Sawyer S, Press WH. 2015. Understanding biases in ribosome profiling experiments reveals signatures of translation dynamics in yeast. PLoS Genet 11: e1005732.

Ingolia NT, Ghaemmaghami S, Newman JRS, Weissman JS. 2009. Genome-wide analysis in vivo of translation with nucleotide resolution using ribosome profiling. Science (New York, NY) 324: 218-223.

Jacobson A, Peltz SW. 1996. Interrelationships of the pathways of mRNA decay and translation in eukaryotic cells. Annu Rev Biochem 65: 693-739.

Jan CH, Williams CC, Weissman JS. 2014. Principles of ER cotranslational translocation revealed by proximity-specific ribosome profiling. Science 346: 1257521.

Johansson M, leong KW, Trobro S, Strazewski P, Aqvist J, Pavlov MY, Ehrenberg M. 2011. pH-sensitivity of the ribosomal peptidyl transfer reaction dependent on the identity of the A-site aminoacyltRNA. Proc Natl Acad Sci 108: 79-84

Lareau LF, Hite DH, Hogan GJ, Brown PO. 2014. Distinct stages of the translation elongation cycle revealed by sequencing ribosomeprotected mRNA fragments. Elife 3: e01257.

Lee SR, Lykke-Andersen J. 2013. Emerging roles for ribonucleoprotein modification and remodeling in controlling RNA fate. Trends Cell Biol 23: 504-510.

Lee M, Wagenmakers E. 2014. Bayesian cognitive modeling: a practical course. Cambridge University Press, Cambridge, UK.

Mishima Y, Tomari Y. 2016. Codon usage and 3' UTR length determine maternal mRNA stability in zebrafish. Mol Cell 61: 874-885. 
Muhlrad D, Parker R. 1992. Mutations affecting stability and deadenylation of the yeast MFA2 transcript. Genes Dev 6: 2100-2111.

Nedialkova DD, Leidel SA. 2015. Optimization of codon translation rates via tRNA modifications maintains proteome integrity. Cell 161: 1606-1618.

Neymotin B, Ettore V, Gresham D. 2016. Multiple transcript properties related to translation affect mRNA degradation rates in Saccharomyces cerevisiae. G3 (Bethesda) 6: 3475-3483.

Pop C, Rouskin S, Ingolia NT, Han L, Phizicky EM, Weissman JS, Koller D. 2014. Causal signals between codon bias, mRNA structure, and the efficiency of translation and elongation. Mol Syst Biol 10: 770.

Presnyak V, Alhusaini N, Chen Y-H, Martin S, Morris N, Kline N, Olson S, Weinberg D, Baker KE, Graveley BR, et al. 2015. Codon optimality is a major determinant of mRNA stability. Cell 160: $1111-1124$

Qian W, Yang JR, Pearson NM, Maclean C, Zhang J. 2012. Balanced codon usage optimizes eukaryotic translational efficiency. PLoS Genet 8: e1002603.

Quax TE, Claassens NJ, Söll D, van der Oost J. 2015. Codon bias as a means to fine-tune gene expression. Mol Cell 59: 149-161.

Radhakrishnan A, Chen YH, Martin S, Alhusaini N, Green R, Coller J. 2016. The DEAD-box protein Dhh1p couples mRNA decay and translation by monitoring codon optimality. Cell 167: 122-132.

Schwarzer G, Carpenter J, Rücker, G. 2015. Meta-analysis with R. Springer, NY.
Shah P, Ding Y, Niemczyk M, Kudla G, Plotkin JB. 2013. Rate-limiting steps in yeast protein translation. Cell 153: 1589-1601.

Smith JE, Alvarez-Dominguez JR, Kline N, Huynh NJ, Geisler S, Hu W, Coller J, Baker KE. 2014. Translation of small open reading frames within unannotated RNA transcripts in Saccharomyces cerevisiae. Cell Rep 7: 1858-1866.

Soneson C, Delorenzi M. 2013. A comparison of methods for differential expression analysis of RNA-seq data. BMC Bioinformatics 14: 91.

Tanner DR, Cariello DA, Woolstenhulme CJ, Broadbent MA, Buskirk AR. 2009. Genetic identification of nascent peptides that induce ribosome stalling. J Biol Chem 284: 34809-34818.

Watts RE, Forster AC. 2010. Chemical models of peptide formation in translation. Biochemistry 49: 2177-2185.

Weinberg DE, Shah P, Eichhorn SW, Hussmann JA, Plotkin JB, Bartel DP. 2016. Improved ribosome-footprint and mRNA measurements provide insights into dynamics and regulation of yeast translation. Cell Rep 14: 1787-1799.

Williams CC, Jan CH, Weissman JS. 2014. Targeting and plasticity of mitochondrial proteins revealed by proximity-specific ribosome profiling. Science 346: 748-751.

Wohlgemuth I, Brenner S, Beringer M, Rodnina MV. 2008. Modulation of the rate of peptidyl transfer on the ribosome by the nature of substrates. J Biol Chem 283: 32229-32235.

Young DJ, Guydosh NR, Zhang F, Hinnebusch AG, Green R. 2015. Rli1/ABCE1 recycles terminating ribosomes and controls translation reinitiation in $3^{\prime} U T R s$ in vivo. Cell 162: 872-884. 